\title{
TIMING AN ACCRETING MILLISECOND PULSAR: MEASURING THE ACCRETION TORQUE IN IGR J00291+5934
}

\author{
L. Burderi, ${ }^{1}$ T. Di Salvo, ${ }^{2}$ G. Lavagetto, ${ }^{2}$ M. T. Menna, ${ }^{3}$ A. Papitto, ${ }^{3,4}$ A. Riggio, ${ }^{2}$ \\ R. IARia, ${ }^{2}$ F. D’Antona, ${ }^{3}$ N. R. Robba, ${ }^{2}$ and L. Stella ${ }^{3}$ \\ Received 2005 August 10; accepted 2006 November 6
}

\begin{abstract}
We performed a timing analysis of the fastest accreting millisecond pulsar IGR J00291+5934 using RXTE data taken during the outburst of 2004 December. We corrected the arrival times of all the events for the orbital (Doppler) effects and performed a timing analysis of the resulting phase delays. In this way we are able to study, for the first time in this class of sources, the spin-up of a millisecond pulsar as a consequence of accretion torques during the X-ray outburst. The accretion torque gives us for the first time an independent estimate of the mass accretion rate onto the neutron star, which can be compared with the observed X-ray luminosity. We also report a revised value of the spin period of the pulsar.
\end{abstract}

Subject headings: pulsars: general — pulsars: individual (IGR J00291+5934) — stars: magnetic fields stars: neutron $-\mathrm{X}$-rays: binaries

\section{INTRODUCTION}

The so-called recycling scenario links two different classes of astronomical objects, namely, the millisecond radio pulsars (usually found in binary systems) and the low-mass X-ray binaries (LMXBs), or at least a subgroup of them. The leading idea of this scenario is the recycling process itself, during which an old, weakly magnetized, slowly spinning neutron star is accelerated by the accretion of matter and angular momentum from a (Keplerian) accretion disk down to spin periods in the millisecond range. In this way, at the end of the accretion phase, the neutron star rotates so fast that it is resurrected from the radio pulsar graveyard, allowing the radio pulsar phenomenon to occur again despite the weakness of the magnetic field.

Although this scenario was first proposed long ago (see, e.g., Bhattacharya \& van den Heuvel 1991 for a review), the most embarrassing problem was the absence of coherent pulsations in LMXBs. Only recently have the long sought millisecond coherent oscillations in LMXBs been found, thanks to the capabilities (the right combination of high temporal resolution and large collecting areas) of the RXTE satellite. In 1998 April a transient LMXB, SAX J1808.4-3658 was discovered to harbor a millisecond pulsar $\left(P_{\text {spin }} \simeq 2.5 \mathrm{~ms}\right)$ in a compact $\left(P_{\text {orb }} \simeq 2 \mathrm{hr}\right)$ binary system (Wijnands \& van der Klis 1998; Chakrabarty \& Morgan 1998). We now know seven accreting millisecond pulsars (Wijnands 2005; Morgan et al. 2005); all of them are X-ray transients in very compact systems (orbital period between 40 minutes and $4 \mathrm{hr})$, the fastest of which $\left(P_{\text {spin }} \simeq 1.7 \mathrm{~ms}\right)$, IGR J00291+5934, was discovered in 2004 December (Galloway et al. 2005, hereafter G05).

Timing techniques applied to data of various accreting millisecond pulsars, spanning the first few days of their outbursts, allowed an accurate determination of their main orbital parameters.

\footnotetext{
${ }^{1}$ Università degli Studi di Cagliari, Dipartimento di Fisica, Monserrato, Italy; burderi@mporzio.astro.it.

2 Dipartimento di Scienze Fisiche ed Astronomiche, Università di Palermo, Palermo, Italy.

3 INAF-Osservatorio Astronomico di Roma, Monteporzio Catone (Rome), Italy.

${ }_{4}^{4}$ Dipartimento di Fisica, Universitá degli Studi di Roma “Tor Vergata”, Rome,
}

However, only a few attempts have been made to determine the spin period derivative (Chakrabarty et al. 2003; Galloway et al. 2002). The first reported measurement of a spin-up in these sources was made for IGR J00291+5934 (Falanga et al. 2005, hereafter F05).

In this paper we apply an accurate timing technique to the fastest currently known accreting millisecond pulsar, IGR J00291+ 5934, with the aim of constraining the predictions of different torque models with good-quality experimental data. Our results indicate quite clearly that a net spin-up occurred during the 2004 December outburst of IGR J00291+5934 (see also F05), and that the derived torque is in good agreement with that expected from matter accreting from a Keplerian disk.

\section{THE TIMING TECHNIQUE}

For a periodic pulsating source, the time of arrival of a given pulse at the solar system barycenter is affected by three effects, which cause temporal delays with respect to the predicted ones. These effects are (1) uncertainties in the orbital parameters, (2) uncertainty in the spin frequency and possible secular variations, and (3) uncertainties in the source position. In standard timing techniques (see, e.g., Blandford \& Teukolsky 1976) the predicted arrival time of a given pulse is computed using a first guess of the parameters of the system, and the difference between the experimental and predicted arrival times, namely the residuals, are fitted with a linear multiple regression of the differential corrections to the parameters. This means that the differential correction to orbital parameters, spin frequency and its derivative, source position in the sky, are computed simultaneously. This technique has the obvious advantage of giving a self-consistent solution, where all the correlations in the covariance matrix of the system are fully taken into account. However, the convergence of the fit is not always guaranteed, and especially in the case of long temporal baselines, convergence to secondary minima might lead to inaccurate solutions.

On the other hand, if the orbital period is much shorter than the timescale on which the spin period derivative and the source position uncertainty are expected to produce a significant effect, it is easy to see that the delays in the arrival times produced by the uncertainties in the orbital parameters are distinguishable from 
those caused by the other two effects. This is because the former oscillate on the orbital period timescale, while it is expected that the latter will follow a secular trend dictated by the torques on the accreting neutron star and by the orbital motion of Earth. In the following sections, we therefore discuss all these effects separately and describe in general the timing analysis suitable for these cases.

The procedure we applied is as follows. In order to obtain the emission times $t_{\mathrm{em}}$, the arrival times of all the events, $t_{\mathrm{arr}}$, were first reported to the solar system barycenter adopting the best estimate of the source position in the sky. We note here that the contribution of the relative acceleration of the binary system with respect to the solar system could in principle produce a nonnegligible contribution to the frequency derivative. For a source located at the position of IGR J00291+5934 at a distance of few $\mathrm{kpc}$, the most relevant of these effects is due to the planar acceleration along the Galactic plane, which gives a spin derivative $\dot{\nu}_{\mathrm{Gal}} \sim 5 \times 10^{-20} \nu$ (see, e.g., Damour \& Taylor 1991). This is several orders of magnitude lower than the spin frequency derivatives we found (see below), and therefore in the following analysis these kind of effects are not taken into account. Then we corrected for the delays caused by the binary motion using the best estimate of the orbital parameters through the (firstorder approximated) formula

$t_{\mathrm{em}} \simeq t_{\mathrm{arr}}-x\left[\sin (m+\omega)+\frac{e}{2} \sin (2 m+\omega)-\frac{3}{2} e \sin \omega\right]$,

where $x=a \sin i / c$ is the projected semimajor axis in light seconds, $m=2 \pi\left(t_{\mathrm{arr}}-T^{*}\right) / P_{\text {orb }}$ is the mean anomaly, $T^{*}$ is the time of ascending node passage at the beginning of the observation, $\omega$ is the periastron angle, and $e$ is the eccentricity. For simplicity, we use $t$ instead of $t_{\mathrm{em}}$. From equation (1) we compute the observed phases as $\phi=\nu\left(t-T_{0}\right)$, where $\nu=1 / P_{\text {spin }}$ and $T_{0}$ is the start time of the observation. On the other hand, the expected phase variations, $\delta \phi_{\dot{\nu}}$, caused by a spin frequency derivative, $\dot{\nu}$, can be computed by a double direct integration

$$
\delta \phi_{\dot{\nu}}(t)=\int_{T_{0}}^{t}\left[\int_{T_{0}}^{t^{\prime}} \dot{\nu}\left(t^{\prime \prime}\right) d t^{\prime \prime}\right] d t^{\prime}
$$

In the simplest case of a constant $\dot{\nu}$, the integration gives a parabolic function of time. In general, fitting these expected phase variations to the observed ones, we can obtain an estimate of $\dot{\nu}(t)$, and hence important information on the torques acting on the accreting neutron star.

To obtain this information it is important to evaluate any source of error in the observed phase variations; we start by discussing the errors induced by the uncertainties on the orbital parameters of the binary system. The differential of $\phi=\nu\left(t-T_{0}\right)$, with $t$ given by equation (1), with respect to the orbital parameters, allows us to calculate the uncertainties in the phases, $\sigma_{\phi, \text { orb }}$, caused by the uncertainties, $\sigma$, in the estimates of the orbital parameters

$$
\begin{aligned}
\sigma_{\phi, \text { orb }} & =\frac{x}{P_{\text {spin }}}\left[\sin ^{2} m\left(\frac{\sigma_{x}}{x}\right)^{2}+\cos ^{2} m\right] \\
& \times\left\{\left[m^{2}\left(\frac{\sigma_{P_{\text {orb }}}}{P_{\text {orb }}}\right)^{2}+\left(\frac{2 \pi \sigma_{T^{*}}}{P_{\text {orb }}}\right)^{2}\right]+\sin ^{2} m \cos ^{2} m \sigma_{e}^{2}\right\}^{1 / 2} .
\end{aligned}
$$

The uncertainties in the adopted orbital parameters will result in a "timing noise" of amplitude $\sigma_{\phi, \text { orb }}$. These therefore should be added in quadrature to the statistical uncertainties $\sigma_{\phi \text {, stat }}$ on the experimentally determined phase delays. The resulting uncertainties on the phase delays will be $\sigma_{\phi}=\left(\sigma_{\phi \text {, orb }}^{2}+\sigma_{\phi \text {, stat }}^{2}\right)^{1 / 2}$.

On the other hand, the uncertainties in the phase delays, $\sigma_{\phi \text {, pos, }}$ caused by the uncertainties in the estimates of the source position in the sky will produce a sinusoidal oscillation in the Earth's orbital period. For observation times shorter than $1 \mathrm{yr}$, as it is the case for most transient accreting millisecond pulsars, this can cause systematic errors in the determination of the neutron star period and its derivative, since a series expansion of a sinusoid contains a linear and a quadratic term. In order to evaluate these effects, let us consider the expression of the phase delays induced by the Earth's motion for a small displacement, $\delta \lambda$ and $\delta \beta$, in the position of the source in ecliptic coordinates $\lambda$ and $\beta$ (see, e.g., Lyne \& Graham-Smith 1990)

$$
\Delta \phi_{\mathrm{pos}}=\nu_{0} y\left[\sin \left(M_{0}+\epsilon\right) \cos \beta \delta \lambda-\cos \left(M_{0}+\epsilon\right) \sin \beta \delta \beta\right],
$$

where $y=r_{\mathrm{E}} / c$ is the distance of the Earth with respect to the solar system barycenter in light seconds, $M_{0}=2 \pi\left(T_{0}-T_{\gamma}\right) / P_{\oplus}-\lambda$, where $T_{\gamma}$ is the time of passage through the vernal point and $\epsilon=2 \pi\left(t-T_{0}\right) / P_{\oplus} \ll 1$ in our case.

After some algebraic manipulations, this can be written as $\Delta \phi_{\text {pos }}=\nu_{0} y \sigma_{\gamma} \sin \left(M_{0}+\epsilon-\theta^{*}\right) u$, where $\sigma_{\gamma}$ is the positional error circle, $\theta^{*}=\arctan (\tan \beta \delta \beta / \delta \lambda)$, and $u=\left[(\cos \beta \delta \lambda)^{2}+\right.$ $\left.(\sin \beta \delta \beta)^{2}\right]^{1 / 2} / \sigma_{\gamma}$. Since the true source position must lie within the error circle, the following inequalities hold: $\left(\cos \beta \delta \lambda / \sigma_{\gamma}\right)^{2} \leq 1$, $\left(\delta \beta / \sigma_{\gamma}\right)^{2} \leq 1$, and thus $u \leq\left(1+\sin ^{2} \beta\right)^{1 / 2}$. This means that the uncertainty in the source position is

$$
\Delta \phi_{\text {pos }} \leq \nu_{0} y \sigma_{\gamma}\left(1+\sin ^{2} \beta\right)^{1 / 2} \sin \left(M_{0}+\epsilon-\theta^{*}\right)
$$

We can expand it in series in the parameter $\epsilon \ll 1$ in order to find the systematic uncertainties induced on the linear and quadratic term. Note that, since the values of $\delta \lambda$ and $\delta \beta$ are the differences between the nominal and the true (unknown) source position (which can be everywhere within the error circle), their ratio is undetermined, and hence $\theta^{*}$ can be any value between 0 and $2 \pi$. We have therefore maximized the functions $\sin \theta^{*}$ and $\cos \theta^{*}$ with 1 separately in the linear and quadratic terms of the series expansion. The resulting systematic error in the linear and the quadratic term of phase delays evolution versus time, which correspond to the spin frequency correction and the spin frequency derivative, respectively, are $\sigma_{\nu \text {, syst }} \leq \nu_{0} y \sigma_{\gamma}\left(1+\sin ^{2} \beta\right)^{1 / 2} 2 \pi / P_{\oplus}$ and $\sigma_{\dot{\nu}, \text { syst }} \leq \nu_{0} y \sigma_{\gamma}\left(1+\sin ^{2} \beta\right)^{1 / 2}\left(2 \pi / P_{\oplus}\right)^{2}$.

Summarizing, the phase variations caused by $\dot{\nu}$ are effectively distinguishable from those induced by the uncertainties in the orbital parameters, which result in a "timing noise" of amplitude given by equation (3). On the other hand, the uncertainty on the source position cannot be easily decoupled from the phase variations caused by $\dot{\nu}$ (particularly for observation times much shorter than $1 \mathrm{yr}$ ) and therefore results in systematic errors on the estimate of the spin frequency and its derivative.

\section{OBSERVATIONS AND DATA ANALYSIS}

IGR J00291+5934 was observed by RXTE between 2004 December 3 and 21. While the observations between December 3 and 6 have already been analyzed in G05, in this paper we analyze the data between December 7 and 21 taken from a public ToO observation. We mainly use data from the RXTE Proportional Counter Array (PCA; Jahoda et al. 1996), which consists of five identical gas-filled proportional counter units (PCUs), 
with a total effective area of $\sim 6000 \mathrm{~cm}^{2}$, sensitive in the energy range between 2 and $60 \mathrm{keV}$. We used data collected in generic Events mode, with a time resolution of $125 \mu$ s and 64 energy channels. These files were processed and analyzed using the FTOOLS v.5.3.1. In order to eliminate the Doppler effects caused by the Earth and satellite motion, the arrival times of all the events were converted to barycentric dynamical times at the solar system barycenter. The position adopted for the source was that of the proposed radio counterpart (which is compatible with that of the proposed optical counterpart; see Rupen et al. 2004; Fox \& Kulkarni 2004). For the spectral analysis we also used data from the High-Energy X-Ray Timing Experiment (HEXTE; Rothschild et al. 1998; 20-200 keV energy range).

We corrected the arrival times of all the events for the delays caused by the binary motion using equation (1) with the orbital parameters given in G05. In order to check for the presence of the pulsations during our observation, we performed an epoch folding search on each continuous interval of data (lasting on average 60 minutes) around the spin period given in G05. The pulsation was clearly visible up to December 12 . After that, owing to poor statistics, pulsations could only be detected by folding $\sim 1$ day worth of data. No pulsations were detected after December 14, in accordance with what is reported in G05.

Using our longer temporal baseline (about 7 days) with respect to that in G05 (about 3 days), we first tried to increase the accuracy of the orbital period measurement using the technique described in Papitto et al. (2005) and successfully applied to SAX J1808.4-3658. However, no significant improvement was found. Adopting the uncertainties in the estimates of the orbital parameters given in G05 in equation (3), we obtained $\sigma_{\phi, \text { orb }} \lesssim$ 0.01 , where we maximized sin and cos functions with 1 , and used $t-T_{0} \lesssim 7$ days. Therefore, we expect that the uncertainties in the orbital parameters will cause a "timing noise" no greater than $\sigma_{\phi, \text { orb }} P_{\text {spin }} \sim 0.02 \mathrm{~ms}$.

To compute phases of good statistical significance, we epoch folded each interval of data in which the pulsation was significantly detected at the spin period given in G05 with respect to the same reference epoch, $T_{0}$, corresponding to the beginning of our observations. The fractional part of the phase was obtained by fitting each pulse profile with a sinusoid of a fixed period. To compute the associated errors we combined the statistical errors derived from the fit, $\sigma_{\phi \text {, stat }}$, with the errors $\sigma_{\phi, \text { orb }}$ as $\sigma_{\phi}=\left(\sigma_{\phi, \text { stat }}^{2}+\sigma_{\phi, \text { orb }}^{2}\right)^{1 / 2}$.

In order to derive the differential correction to the spin frequency $\Delta \nu_{0}$ and its derivative $\dot{\nu}_{0}$ at the time $T_{0}$, we had to derive a functional form for the time dependence of the phase delays. We started from the simple assumptions briefly summarized below.

1. The bolometric luminosity $L$ is a good tracer of the mass accretion rate $\dot{M}$ via the relation $L=\zeta(G M / R) \dot{M}$, where $\zeta \leq 1$, and $G, M$, and $R$ are the gravitational constant and the neutron star mass and radius, respectively.

2. The matter accretes through a Keplerian disk truncated at the magnetospheric radius, $R_{m} \propto \dot{M}^{-\alpha}$, by its interaction with the (dipolar) magnetic field of the neutron star. At $R_{m}$ the matter is forced to corotate with the magnetic field of the neutron star and is funneled (at least in part) toward the rotating magnetic poles, thus causing the pulsed emission. For standard disk accretion $\alpha=2 / 7$; note that this is very close to the upper limit $\alpha=2.3 / 7$ derived from our data. This upper limit on $\alpha$ can be derived by noting that the pulsations that were clearly seen at the beginning of the observations in G05 at MJD 53342.0 and were detected for $\Delta t \sim 11.5$ days until MJD $\sim 53353.5$, when the flux reduced by a factor of $\sim 10$. The corresponding expansion in $R_{m}$ must be $R_{\max } / R_{\min }=\left(1-\Delta t / t_{B}^{*}\right)^{-\alpha} \leq R_{\mathrm{CO}} / R$, which for $R=10^{6} \mathrm{~cm}$ and $m=1.4$ gives $\alpha \leq \alpha_{\max }=0.328 \simeq 2.3 / 7$, where $t_{B}^{*} \sim 12.4$ days is the decay time from $\mathrm{G} 05$ and $R_{\mathrm{CO}}$ is the corotation radius, both defined below.

Therefore, we considered two extreme cases, namely $\alpha=2 / 7$ and $\alpha=0$, since a location of $R_{m}$ independent of $\dot{M}$ has been proposed (see, e.g., Rappaport et al. 2004).

3 . The matter accretes onto the neutron star its specific Keplerian angular momentum at $R_{m}, \ell=\left(G M R_{m}\right)^{1 / 2}$, thus causing a material torque $\tau_{\dot{M}}=\ell \dot{M}$. A firm upper limit to this torque is given by the condition $\tau_{\dot{M}} \leq \ell_{\max } \dot{M}$, with $\ell_{\max }=\left(G M R_{\mathrm{CO}}\right)^{1 / 2}$, where $R_{\mathrm{CO}}=1.50 \times 10^{8} \mathrm{~m}^{1 / 3} \mathrm{\nu}^{-2 / 3}$ is the corotation radius (namely the radius at which the Keplerian frequency equals $\nu$ and beyond which accretion is centrifugally inhibited), and $m=M / M_{\odot}$.

4. We do not consider any form of threading of the accretion disk by the magnetic field of the neutron star (see, e.g., Ghosh \& Lamb 1979; Wang 1997, 1996; Rappaport et al. 2004 for a description of the magnetic threading), which implies that the only torque acting during accretion is $\tau_{\dot{M}}$.

Under these hypotheses, the spin frequency derivative is $\dot{\nu}=$ $\ell \dot{M} /(2 \pi I)$, where $I$ is the moment of inertia of the neutron star and we have neglected any variation of $I$ caused by accretion. If $\dot{M}=\dot{M}(t)$, we have $\dot{\nu}(t)=(2 \pi I)^{-1} \ell_{0} \dot{M}_{0}\left[\dot{M}(t) / \dot{M}_{0}\right]^{1-\alpha / 2}$, where $\ell_{0}=\left(G M R_{m, 0}\right)^{1 / 2}$, and $R_{m, 0}$ and $\dot{M}_{0}$ are $R_{m}$ and $\dot{M}$ at $t=T_{0}$, respectively. For the $\alpha=0$ case we assumed $\ell_{0}=\ell_{\max }$. In this case we therefore assume that the system is accreting the maximum specific angular momentum possible, giving an upper limit on the spin-up torque and therefore a lower limit to the accretion rate $\dot{M}_{0}$.

Since we assume $\dot{M}(t) \propto L(t)$, to determine the temporal dependence of $\dot{M}(t)$ we studied the energy spectra of the source for each continuous interval of data, combining the PCA and HEXTE data. All the spectra are well fitted with a model consisting of a power law with an exponential cutoff plus thermal emission from a Keplerian accretion disk modified by photoelectric absorption and a Gaussian iron line. In order to derive $L(t)$ for each spectrum we made the simple assumption $L(t) \propto$ $F_{(3-150)}(t)$, which is the unabsorbed flux in the RXTE PCA plus HEXTE energy band (3-150) keV. A good fit of $F_{(3-150)}(t)$ versus $t$ between December 7 and 14 ( $\Delta t_{\mathrm{obs}} \sim 7$ days) is given by the expression $F_{(3-150)}(t)=F_{(3-150)}\left[1-\left(t-T_{0}\right) / t_{B}\right]$, with $t_{B}=$ $8.4 \pm 0.1$ days, where $F_{(3-150)}$ is the unabsorbed flux at $t=T_{0}$. Therefore, we have $\dot{\nu}(t)=\dot{\nu}_{0}\left[1-\left(t-T_{0}\right) / t_{B}\right]^{1-\alpha / 2}$, where the spin frequency derivative at $t=T_{0}$ is $\dot{\nu}_{0}=(2 \pi I)^{-1} \ell_{0} \dot{M}_{0}$.

With this expression for $\nu(t)$, equation (2) can be readily integrated. Since $\epsilon=\left(t-T_{0}\right) / t_{B}<1$ for $t<7$ days, we took a series expansion of the integral and obtained $\delta \phi_{\dot{\nu}}(t)=1 / 2 \dot{\nu}_{0}\left(t-T_{0}\right)^{2}[1-$ $\left.(2-\alpha)\left(t-T_{0}\right) /\left(6 t_{B}\right)+\xi\right]$, with an error $\xi<\alpha(1-\alpha / 2) / 24 \epsilon^{2}$.

We have therefore fitted these phases with the function

$$
\phi=-\phi_{0}-\Delta \nu_{0}\left(t-T_{0}\right)-\frac{1}{2} \dot{\nu}_{0}\left(t-T_{0}\right)^{2}\left[1-\frac{(2-\alpha)\left(t-T_{0}\right)}{6 t_{B}}\right] \text {. }
$$

Using the best-fit value for $\Delta \nu_{0}$, we computed the improved spin frequency estimate and repeated the same procedure described at the beginning of this paragraph, folding at the new estimate of the spin period. The new phases were fitted with equation (6). In this case, $\Delta \nu_{0}$ was fully compatible with zero. These phases are plotted versus time in Figure 1 (top panel) together with the residuals in units of $\sigma$ with respect to equation (6) (bottom panel). The best-fit estimates of $\nu_{0}$ and $\dot{\nu}_{0}$ are reported in Table 1 


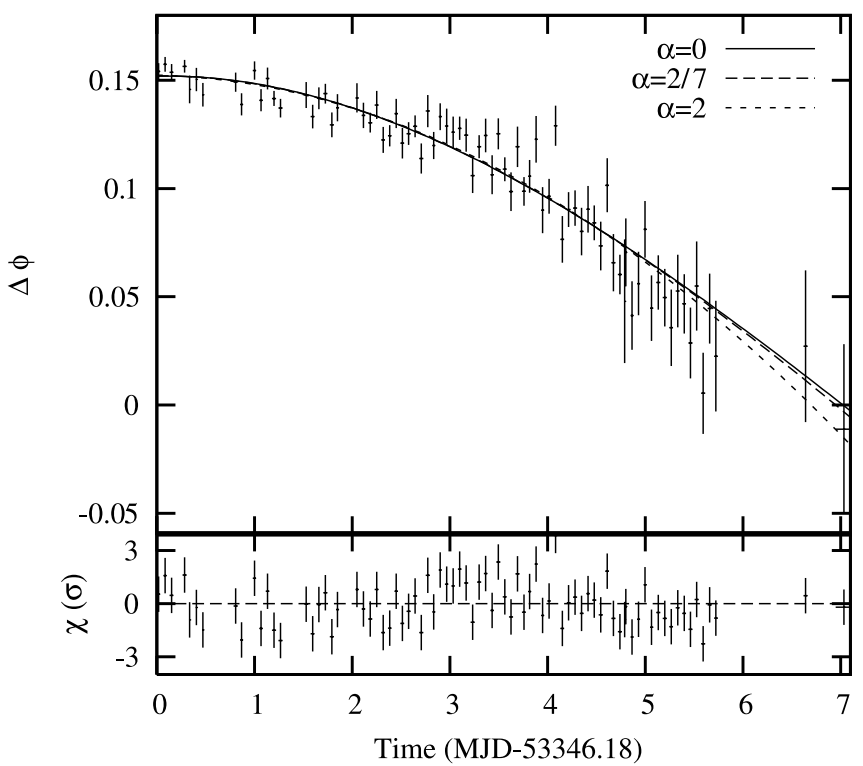

Fig. 1.-Pulse phases computed by folding at the spin period reported in Table 1 and plotted vs. time together with the best-fit curves (top) and residuals in units of $\sigma$ with respect to the model with $\alpha=2 / 7$ (bottom).

for three values of $\alpha: \alpha=0$, which correspond to a location of $R_{m}$ independent of the accretion rate (cf. the model of Rappaport et al. 2004 in which $R_{m}=R_{\mathrm{CO}}$ for any $\dot{M}$ ); the standard case $\alpha=$ $2 / 7$, which corresponds to $R_{m}$ proportional to the Alfvén radius; and $\alpha=2$, which has been given for comparison purposes and corresponds to a parabolic trend, expected in the case of constant $\dot{M}$. Of course, the value of $\dot{\nu}$ obtained in this latter case is in agreement with the value obtained by F05 of $\dot{\nu}=8.4(6) \times$ $10^{-13} \mathrm{~Hz} \mathrm{~s}^{-1}$. A comparison of the $\chi^{2} /$ dof for each of the adopted values of $\alpha$ (also reported in Table 1) shows that the statistics are not good enough to distinguish between these three possibilities.

Finally, to evaluate the systematic errors on the spin frequency and its derivative, we adopted the positional uncertainty of $0.06^{\prime \prime}$ radius reported by Rupen et al. (2004) in a series expansion of equation (5), finding $\sigma_{\nu \text {, syst }} \sim 2.2 \times 10^{-8} \mathrm{~Hz}$ and $\sigma_{\dot{\nu} \text {,syst }} \sim$ $4.4 \times 10^{-15} \mathrm{~Hz} \mathrm{~s}^{-1}$, respectively. Even adopting a positional error circle of $0.2^{\prime \prime}$ (which is the distance between the optical and radio position; Fox \& Kulkarni 2004), the resulting sys- tematic uncertainties are $\sigma_{\nu \text {,syst }} \sim 7.3 \times 10^{-8} \mathrm{~Hz}$ and $\sigma_{\dot{\nu} \text {, syst }} \sim$ $1.5 \times 10^{-14} \mathrm{~Hz} \mathrm{~s}^{-1}$. Note that the systematic error on the spin frequency is comparable with the error derived from the fit of the phase delays and reported in Table 1, the systematic error on the spin frequency derivative is at least 1 order of magnitude below the error derived from the fit.

\section{DISCUSSION}

From the best-fit value of the spin frequency derivative $\dot{\nu}_{0}$ we can compute the mass accretion rate at $t=T_{0}$ through the formula

$$
\dot{M}_{-10}=5.9 \dot{\nu}_{-13} I_{45} m^{-2 / 3}\left(R_{\mathrm{CO}} / R_{m, 0}\right)^{1 / 2},
$$

where $\dot{M}_{-10}$ is $\dot{M}_{0}$ in units of $10^{-10} M_{\odot} \mathrm{yr}^{-1}, \dot{\nu}_{-13}$ is $\dot{\nu}_{0}$ in units of $10^{-13} \mathrm{~s}^{-2}$, and $I_{45}$ is $I$ in units of $10^{45} \mathrm{~g} \mathrm{~cm}^{2}$. In the following discussion we adopt the FPS equation of state for the neutron star matter for $m=1.4$ and the spin frequency of IGR J00291+ 5934, which gives $I_{45}=1.29$ and $R=1.14 \times 10^{6} \mathrm{~cm}$ (see, e.g., Cook et al. 1994). This gives a lower limit in the mass accretion rate of $\dot{M}_{-10} \sim 70 \pm 10$ (case $\alpha=0$ ). In order to compare the experimental estimate of $\dot{M}_{0}$ with the observed X-ray luminosity, we have to derive the bolometric luminosity $L(t)$ from the observed flux $F_{(3-150)}(t)$. To this end we consider the spectral shape at $t=T_{0}$ in more detail.

Since the value of the hydrogen column $N_{\mathrm{H}}$ is poorly constrained in the RXTE energy band that starts at $2.5 \mathrm{keV}$, we fixed it to $N_{\mathrm{H}}=2.80 \times 10^{21} \mathrm{~cm}^{-2}$, which is the value obtained by Nowak et al. (2004) analyzing the Chandra data. Our spectral results are practically independent of the precise value of the $N_{\mathrm{H}}$ below the total Galactic hydrogen column in the direction of IGR J00291+5934.

The power law is the dominant spectral component. In particular, we found a power-law spectral index $\alpha=-0.59_{-0.034}^{+0.047}$ and an $e$-folding energy $E_{\text {fold }} \sim 178_{-47}^{+83} \mathrm{keV}$ (with values ranging from 60 to over $260 \mathrm{keV}$ throughout our data). The ratio of the unabsorbed fluxes in the bands $0.001-1000 \mathrm{keV}$ and $3-150 \mathrm{keV}$ is 1.6. This ratio is almost independent on the $e$-folding energy, increasing up to $8 \%$ when the $e$-folding energy increases from 60 to $260 \mathrm{keV}$. Therefore, we assume $F_{\mathrm{PL}(0, \infty)} \simeq 1.6 F_{\mathrm{PL}(3-150)}$. The power-law component presumably originates in regions of small optical depth just above each polar cap (see, e.g., Poutanen \& Gierlinski 2003; Gierlinski \& Poutanen 2005), thus we neglect to the first order any effect of the inclination of the emitting region

TABLE 1

Orbital and Spin Parameters of IGR J00291+5934

\begin{tabular}{|c|c|c|c|}
\hline Parameter & G05 & F05 & This Work \\
\hline Orbital period, $P_{\text {orb }}(\mathrm{s}) \ldots$ & $8844.092(6)$ & $\ldots$ & $\ldots$ \\
\hline Eccentricity, $e$ & $<2 \times 10^{-4}(3 \sigma)$ & $\ldots$ & $\ldots$ \\
\hline Spin frequency, $\nu_{0}(\mathrm{~Hz})$ & $598.89213064(1)$ & $598.89213060(1)$ & $598.89213053(2)$ \\
\hline 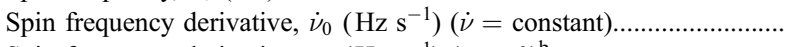 & $<8 \times 10^{-13}(3 \sigma)$ & $8.4(6) \times 10^{-13}$ & $0.85(0.11) \times 10^{-12}\left(\chi^{2} / \mathrm{dof}=106 / 77\right)$ \\
\hline 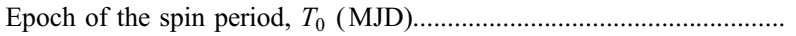 & $\ldots$ & 53346.0 & 53346.184635 \\
\hline
\end{tabular}

NotEs.-Errors are given at $1 \sigma$ confidence level. The errors quoted for the spin frequency and spin frequency derivative are derived from the phase delays fitting and

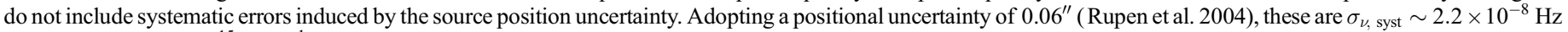
and $\sigma_{\dot{\nu}, \text { syst }} \sim 4.4 \times 10^{-15} \mathrm{~Hz} \mathrm{~s}^{-1}$, respectively.

${ }^{a}$ G05 reported a value of 53345.1875164(4) MJD for the epoch of superior conjunction, i.e., when the neutron star is behind the companion, as in this work, we considered the epoch of ascending node passage as a reference time, the G05 reference time reported here has been decremented by $P_{\text {orb }} / 4$.

$\mathrm{b}$ Averaging $\dot{\nu}_{0}$ over the 7 days of our observation we get $\left\langle\dot{\nu}_{0}\right\rangle \simeq 0.68 \times 10^{-12} \mathrm{~Hz} \mathrm{~s}^{-1}$. 
with respect to the observer. On the other hand, we observe a single-peaked pulse profile, which means that we only see the emission from one of these regions (e.g., Kulkarni \& Romanova 2005).

If this is the case, we have to take into account the possibility that we are underestimating the total flux in the power-law component because one of the two polar caps is never visible (this can happen if the sum of the angles between the magnetic axis and spin axis and between the line of sight and the spin axis is less than $\pi / 2$ ). If we only see polar cap A, we indicate it with $F_{\mathrm{A}}$, the flux emitted by A reaching the observer. Because the emission from $\mathrm{A}$ is isotropic, to obtain the luminosity we have to integrate over $4 \pi d^{2}$, so we obtain $L_{\mathrm{A}}=F_{\mathrm{A}} 4 \pi d^{2}$. However, under our assumptions, this is an underestimate of the total $(\mathrm{A}+\mathrm{B})$ luminosity of the system consisting of the two polar caps, since part of the flux $\left(F_{\mathrm{B}}\right)$ that should be emitted in the direction of the observer is never visible.

Actually, almost half of the emission from cap A facing the neutron star surface is intercepted by the neutron star itself and reemitted toward the observer, and this reemission should indeed be considered. However, it is reasonable to assume that this intercepted emission is reprocessed by the neutron star and reemitted as a blackbody-like spectrum at a relatively low temperature. This reprocessed emission therefore has a very different spectral shape from the original power-law component; simple estimates demonstrate that the temperature associated with this blackbody-like emission is below $1 \mathrm{keV}$, and thus not related to the power-law component. Moreover, most of the emission from this component is outside the energy range of $R X T E / \mathrm{PCA}$ (and thus poorly constrained by the $R X T E$ observation).

Since the emission from an optically thin region is proportional to the volume of the emitting region visible, in the hypothesis that we totally miss the flux from one of the two polar caps, we should multiply the unabsorbed flux of the power law by a factor of 2 in order to take into account the emission of the optically thin region above the unseen polar cap. We therefore parameterize the luminosity of the power-law component with a factor $\eta$ (which can assume values between 1 and 2), and we can write the total luminosity of the two polar caps as $L_{\mathrm{PL}} \simeq$ $\eta F_{\mathrm{PL}(0, \infty)} 4 \pi d^{2}=0.75_{-0.15}^{+0.20} \times 10^{37} \eta d_{5 \mathrm{kpc}}^{2}$ ergs s${ }^{-1}$, where $d_{5 \mathrm{kpc}}$ is the source distance in units of $5 \mathrm{kpc}$. The uncertainty on the luminosity has been evaluated conservatively by propagating the uncertainties on the spectral parameters treated as if they were independent of each other.

The second component is the thermal emission interpreted as emission from a Shakura-Sunyaev accretion disk, which is fitted with the diskbb model. We found a temperature of the inner rim of the accretion disk of $T_{\text {in }}=0.68_{-0.25}^{+0.20} \mathrm{keV}$. On the other hand, because of the poor coverage of $R X T E / \mathrm{PCA}$ at soft X-rays, the disk blackbody normalization $K=\left(R_{\mathrm{in}, \mathrm{km}} / D_{10 \mathrm{kpc}}\right)^{2} \cos \theta=25_{-20}^{+170}$ (where $D_{10} \mathrm{kpc}$ is the distance in units of $10 \mathrm{kpc}$ ) is basically unconstrained. We therefore use the inner disk temperature and the virial theorem to infer the bolometric luminosity of the disk as follows. We interpret the inner temperature of the accretion disk as derived by the diskbb model as the maximum temperature in the disk. To prove this we have also fitted the soft X-ray spectrum with the diskpn model (instead of the diskbb model); diskpn takes into account corrections for temperature distribution near the compact object using the Paczynski-Wiita pseudoNewtonian potential (see Gierlinski et al. 1999). This model also gives a maximum temperature of the disk of $0.70 \mathrm{keV}$ (fully compatible with the value obtained with the diskbb model). Standard disk theory (see, e.g., Frank et al. 2002) predicts that the temperature of the disk attains a maximum value at a radius of $49 / 36 R_{*}$, where $R_{*}$ is the radius at which the disk is truncated, corresponding to $R_{m, 0}$ in our case. Using this in equation (5.43) of Frank et al. (2002) we obtain the inner disk radius through the relation $R_{m, 0}=$ $1.77 m^{1 / 3} \dot{M}_{-10}^{1 / 3} T_{\mathrm{keV}}^{-4 / 3} \mathrm{~km}$. Combining this with equation (7) and adopting $T_{\mathrm{keV}}=0.70$, we solve for the mass accretion rate and the inner disk radius. We found a mass accretion rate $\dot{M}_{-10}=$ $85 \pm 19$ and an inner disk radius $R_{m, 0} \simeq 1.46_{-0.49}^{+0.62} \times 10^{6} \mathrm{~cm}$, which is exactly within the very narrow range between the neutron star radius $\left(\sim 10^{6} \mathrm{~cm}\right)$ and the corotation radius $(\sim 2.4 \times$ $\left.10^{6} \mathrm{~cm}\right)$; the agreement with the expectation is compelling. The virial theorem allows to calculate the fraction of the total luminosity that is emitted by the disk as $0.5 R / R_{m, 0}=0.39$. Therefore, $L_{\mathrm{BB}, 0}=0.39 /(1-0.39) L_{\mathrm{PL}, 0}=4.8 \times 10^{36} \eta d_{5 \mathrm{kpc}}^{2} \mathrm{ergs} \mathrm{s}^{-1}$.

The total bolometric luminosity is therefore $L_{0}=1.23_{-0.15}^{+0.45} \times$ $10^{37} \eta d_{5 \mathrm{kpc}}^{2} \operatorname{ergs~}^{-1}$. If we compare this luminosity with the mass accretion rate inferred from the timing analysis (assuming an efficiency $\zeta=1$ ), we obtain a distance to the source in the range $(10.5-15) / \eta^{1 / 2} \mathrm{kpc}$. Note that $10 \mathrm{kpc}$ is close to the edge of our Galaxy in the direction of IGR J00291+5934. If we push the factor $\eta$ to its maximum value of 2 , we obtain a more reasonable range of distances to the source of 7.4-10.7 kpc, consistent with the lower limit of $5 \mathrm{kpc}$ discussed in F05. Note also that the effect of including magnetic torques due to threading (see assumption [4] in $\S 3$ ) can only push the source further away. This is evident from the $\alpha=0$ case (which gives a spin derivative similar to the $\alpha=2 / 7$ case discussed here) in which we assume that the system is accreting the specific angular momentum at the corotation radius, which is the maximum possible, since any torque beyond the corotation could only spin down the system, this would increase the required $\dot{M}$ to justify the measured spin-up.

Finally, using the value of the inner disk radius derived above and the normalization of the disk blackbody model, $K=$ $\left(R_{\mathrm{km}} / D_{10 \mathrm{kpc}}\right)^{2} \cos \theta=25_{-20}^{+170}$, we infer the inclination of the system with respect to the line of sight, which is $i \geq 40^{\circ}$ for a distance of $9.5 \mathrm{kpc}$.

\section{CONCLUSIONS}

We have analyzed $R X T E$ data of the fastest known accreting millisecond pulsar, IGR J00291+5934, during the period 2004 December 7-14. We report a revised estimate of the spin period and discuss the spin period derivative. The source shows a strong spin-up, which indicates a mass accretion rate of about $8.5 \times$ $10^{-9} M_{\odot} \mathrm{yr}^{-1}$. We have checked that this mass accretion rate is compatible with the X-ray spectrum of the source. In particular we have shown that, with this high accretion rate, the measured temperature of the disk blackbody emission implies an inner disk radius in excellent agreement with the inferred magnetospheric radius of the source [constrained to be in the narrow range $\left.(1-2) \times 10^{6} \mathrm{~cm}\right]$, and that the source is probably seen at high inclination. However, the mass accretion rate inferred by the observed spin-up (and calculated using standard values for the neutron star moment of inertia), using the simple relation $L_{\text {bol }}=\zeta G M \dot{M} / R$ (with an efficiency $\zeta=1$ ), would correspond to quite a high bolometric luminosity of the source of $L_{\mathrm{bol}} \sim$ $10^{38} \mathrm{ergs} \mathrm{s}^{-1}$, much higher than the observed source luminosity assuming a distance of $5 \mathrm{kpc}$. We propose that the simplest explanation of this discrepancy is that part of the accretion luminosity is not visible. Indeed, if we only see the emission of one of the two polar caps, we could miss up to half of the flux in the power-law component. Under this hypothesis (described in detail in $\S 4$ ), we have extrapolated the flux of the source derived from the X-ray spectrum, which corresponds to a bolometric luminosity of $L_{\mathrm{bol}} \simeq 2.5 \times 10^{37} d_{5 \mathrm{kpc}}^{2} \mathrm{ergs} \mathrm{s}^{-1}$. Comparing this 
extrapolated bolometric luminosity with the mass accretion rate of the source as derived from the timing, we find an agreement between these two quantities if we place the source at a distance between 7 and $10 \mathrm{kpc}$.

Other possibilities to explain this discrepancy can be that part of the accretion luminosity is not observed because it is emitted in other energy bands, or because the efficiency $\zeta$ of the conversion of the gravitational potential energy of the accreting matter into X-ray luminosity is less than 1 , or because of occultation effects (which may be favored if indeed the source is highly inclined). In these cases we should conclude that the observed X-ray luminosity is not a good tracer of the total mass accretion rate $\dot{M}$ onto the neutron star.

This work was partially supported by the Ministero della Istruzione, della Università e della Ricerca (MIUR).
Bhattacharya, D., \& van den Heuvel, E. P. J. 1991, Phys. Rep., 203, 1

Blandford, R., \& Teukolsky, S. A. 1976, ApJ, 205, 580

Chakrabarty, D., \& Morgan, E. H. 1998, Nature, 394, 346

Chakrabarty, D., et al. 2003, Nature, 424, 42

Cook, G. B., Shapiro, S. L., \& Teukolsky, S. A. 1994, ApJ, 424, 823

Damour, T., \& Taylor, J. H. 1991, ApJ, 366, 501

Falanga, M., et al. 2005, A\&A, 444, 15 (F05)

Fox, D. B., \& Kulkarni, S. R. 2004, Astron. Tel., 354

Frank, J., King, A., \& Raine, D. J. 2002, Accretion Power in Astrophysics (3rd ed.; Cambridge: Cambridge Univ. Press)

Galloway, D. K., Chakrabarty, D., Morgan, E. H., \& Remillard, R. A. 2002, ApJ, 576, L137

Galloway, D. K., Markwardt, C. B., Morgan, E. H., Chakrabarty, D., \& Strohmayer, T. E. 2005, ApJ, 622, L45 (G05)

Ghosh, P., \& Lamb, F. K. 1979, ApJ, 234, 296

Gierlinski, M., \& Poutanen, J. 2005, MNRAS, 359, 1261

Gierlinski, M., Zdziarski, A. A., Poutanen, J., Coppi, P. S., Ebisawa, K., \& Johnson, W. N. 1999, MNRAS, 309, 496

\section{REFERENCES}

Jahoda, K., Swank, J. H., Giles, A. B., Stark, M. J., Strohmayer, T., Zhang, W., \& Morgan, E. H. 1996, Proc. SPIE, 2808, 59

Kulkarni, A. K., \& Romanova, M. M. 2005, ApJ, 633, 349

Lyne, A. G., \& Graham-Smith, F. 1990, Pulsar Astronomy (Cambridge: Cambridge Univ. Press)

Morgan, E., Kaaret, P., \& Vanderspeck, R. 2005, Astron. Tel., 523

Nowak, M. A., et al. 2004, Astron. Tel., 369, 1

Papitto, A., Menna, M. T., Buderi, L., Di Salvo, T., D’Antona, F., \& Robba, N. R. 2005, ApJ, 621, L113

Poutanen, J., \& Gierlinski, M. 2003, MNRAS, 343, 1301

Rappaport, S. A., Fregeau, J. M., \& Spruit, H. 2004, ApJ, 606, 436

Rothschild R. E., et al. 1998, ApJ, 496, 538

Rupen, M. P., Dhawan, V., \& Mioduszewski, A. J. 2004, Astron. Tel., 364, 1

Wang, Y.-M. 1996, ApJ, 465, L111 1997, ApJ, 475, L135

Wijnands, R. 2007, in Pulsars New Research (New York: Nova Science), in press (astro-ph/0501264)

Wijnands, R., \& van der Klis, M. 1998, Nature, 394, 344 